DOI: http://dx.doi.org/10.14311/TPFM.2016.023

\title{
RICHTMYER-MESHKOV AND RAYLEIGH-TAYLOR FRACTAL MIXING
}

\author{
J. M. Redondo ${ }^{1,2}$, T. Vila ${ }^{2}$, J. Tellez ${ }^{1}$, P. L. Gonzalez-Nieto ${ }^{2,3}$ and M. A. Sanchez ${ }^{1,2}$ \\ ${ }^{1}$ Department of Physics, UPC-Barcelona Tech. Universitat Politecnica de Cataluña, \\ Campus Nord B5, 08034, Spain. \\ ${ }^{2}$ Pan European Laboratory on Non-Homogeneous Turbulence, ERCOFTAC, Instituto \\ Pluri Disciplinar, Juan XXIII, 1, Madrid, Spain \\ ${ }^{3}$ Dept. Mathematics, Faculty of Biology, Ciudad Universitaria, Madrid, Spain.
}

\begin{abstract}
Turbulence affects molecular mixing in a large variety of physical processes both in the environment, in astrophysics and in industrial situations. In some events it is interesting to enhance the transport of mass, heat, humidity and pollutants, while sometimes it is interesting to reduce mixing. Here we analyse some turbulent descriptors which reflect the mixing processes in the compressible induced instabilities that take place in shocks, such as Richtmyer-Meshkov and Rayleigh-Taylor (RM and RT). We present results related to both instabilities and discuss their spatial and temporal variability during the advance of a mixing front, and also their relationships with other scaling arguments. Two types of experiments were used in this study: Mixing generated by gravitational acceleration in low Atwood number incompressible experiments using fluids; and the full compressible shocktube experiments using interfaces between different density gases. The role of local turbulence has mostly relied on advanced visualization measurements through multifractal methods. Comparisons with numerical experiments of shock driven fronts occurring at density interfaces are also relevant. The global advance of the fronts is also measured and fractal descriptors are calculated in both Large Eddy Simulation (LES) and Kinematic Simulation KS models.
\end{abstract}

Keywords: mixing, Rayleigh-Taylor, Richtmyer-Meshkov, instabilities, fractal scaling, shock induced turbulence

\section{Introduction}

As part of an international ISTC Project on Neural-Network for Turbulent Mixing, a large number of different experiments were compared using advanced visualization to jointly analyse the Fractal and Multi-scale instabilities leading to mixing due to Rayleigh-Taylor and Richtmeyer-Meshkov instabilities. Using also wavelet and Neural techniques was an additional way of comparing the mixing flows [1-5].

We present turbulence analysis and applications of Fractal analysis in Shock induced compressible and incompressible flows in order to compare complex mixing processes. The applications of advanced multifractal methods in order to evaluate the scale to scale transfer of energy and other descriptors of great importance in mixing processes are difficult to use in combination with other methods [6-8]. In particular we present the evolution of fluxes as molecular mixing takes place, here the use of fast reactive indicators such as Phenoftalein, which provides visual indication of the complexity of shock and buoyancy driven flows. Surface Flow image velocimetry (SFIV) as discussed in [9-11], this allows to measure complex surface velocity fields in engineering involving 3D flow-boundary conditions and complex divergence and wave prone non uniform flows and boundary layer interactions, this may be particularly important when a single camera for PIV provides a 2D image of the real complex flows [10]. The use of multifractal analysis and improvements on Structure function calculations on standard PIV, and on several methods used in experimental fluids mechanics, calibrated towards the understanding of molecular mixing and the role of vorticity and helicity in the analysis of velocity vectors, the divergence and vorticity-stream function parameter spaces in stirring and mixing, seem very important. The spectral behavior has an important role in mixing processes, here we present a practical application in order to present the methodology that we have developed for the analysis of shock tube membrane analysis in collaboration with the DEA and the Department of Engineering of Marseille University (ISTV, St. Jerome) with the Physics Department of the UPC working on the measurement of a shock tube marked by LIF or Schlieren (Figures 1, 2) [7 - 10], as well as in other Fractal Flows. 

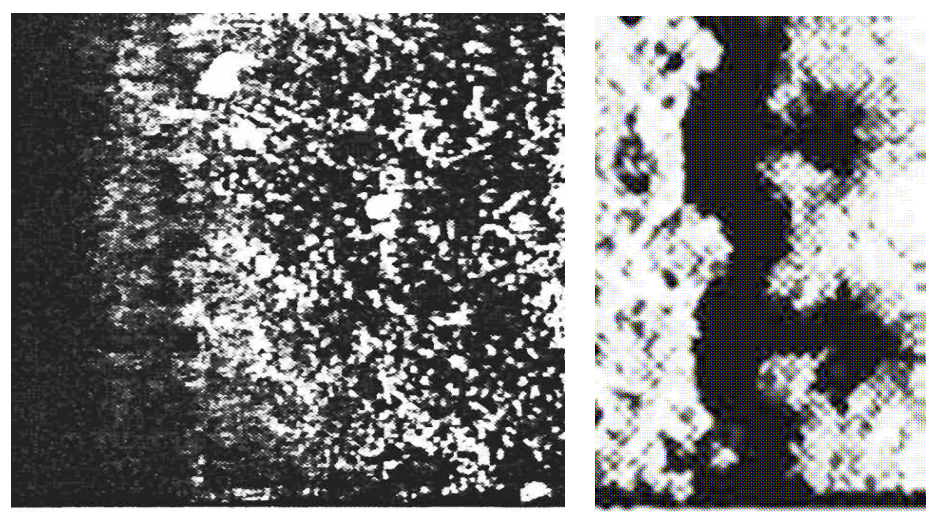

Figure 1: Detail of the mixing front in a Shock tube RM.

Marseille large facility DEA-UPC. (Redondo 1997)
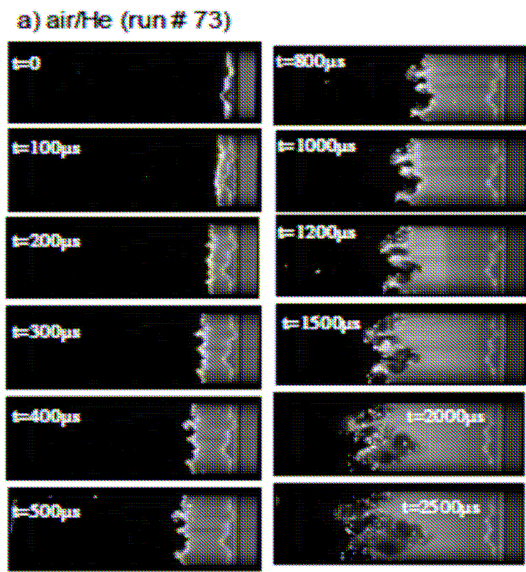

b) airrSF6 (run \# 74)

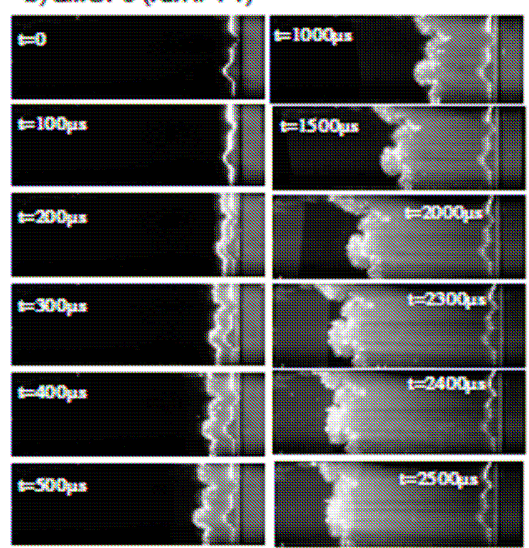

Figure 2: LIF image of the details of the evolution of two RM Shock front experiments [4,5]

\section{Experimental and Numerical Results}

The Richtmyer-Meshkov instability derives its name from the pioneering work of Robert D. Richtmyer at Los Alamos National Laboratory (LANL) who performed the first instability analysis, and Evgeny E. Meshkov at the Russian Federal Nuclear Center-All-Russian Research Institute of Experimental Physics (RFNC-VNIIEF) who performed the first experiments to study this instability". The misalignment of the pressure gradient from the shock wave with the density gradient at the interface produces the vorticity distribution shown in figure 2, which has opposite sign depending on the density gradient direction. Although the shock interaction process is inherently compressible, Richtmyer-Meshkov instability can also be produced by the impulsive acceleration of two incompressible fluids. In this sense, RichtmyerMeshkov instability is related to the better known Rayleigh-Taylor instability [6], and is often referred to as impulsive Rayleigh-Taylor instability. The physical mechanism producing the instability is easily done [8] with a sudden acceleration stop of a tank in a falling frame of reference such as vertical railings.

Obtaining velocity and vorticity fields for these types of flows is made using existing methods based on a technique such as Surface Flow Image Velocimetry (SFIV) similar to the Particle Image Velocimetry (PIV) or laser anemometry, which may be used without the need of adding tracer particles to the flow, but focusing in the monitoring the shapes of the vortical or wave flows. For the application of S.F.I.V. local capillary waves, vortices or wakes as in the case of surface flow. Very high resolution cameras with high frame speed, with large computer capacity and the implementation of programs coupled to advanced image processing for fluid mechanics, such as ImaCalc, DigImage and DigiFlow are necessary [8-11]. We can resolve 2D velocities in a LIF plane or image sequence as a general laboratory tool. In mixing produced by either Rayleigh-Taylor instability, Richtmyer-Meshkov Instability or Shear KelvinHelmholtz billows. Let us recall that Rayleigh-Taylor instability occurs when two fluids, either miscible 
or not miscible are initially placed on top of each other with the heaviest one at the top; if the viscosities are small enough, then the two fluids get mixed without interpenetrating each other and develop mushroom-type structures that degenerate into extremely thin and twisted filaments, see [12-16].

There is uncertainty is defining the 'size' of a turbulent eddy, a problem inherent to the nature of turbulence. Consequently, it is not possible to determine the precise cuts between scaling ranges. This may appear to be a difficult problem. But we remark that such uncertainty is a characteristic of turbulence and is implicit even in Richardson's locality hypothesis where a lack of precise definition of the size of a local eddy does not prevent scaling laws from being obtained [16-18].

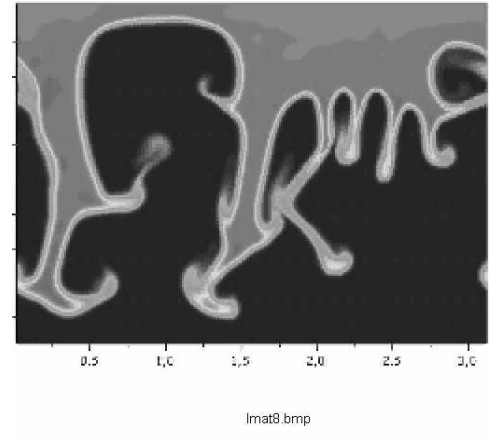

Figure 3: RT instability at $\mathrm{t} / \mathrm{T}=2$ of LES simulation

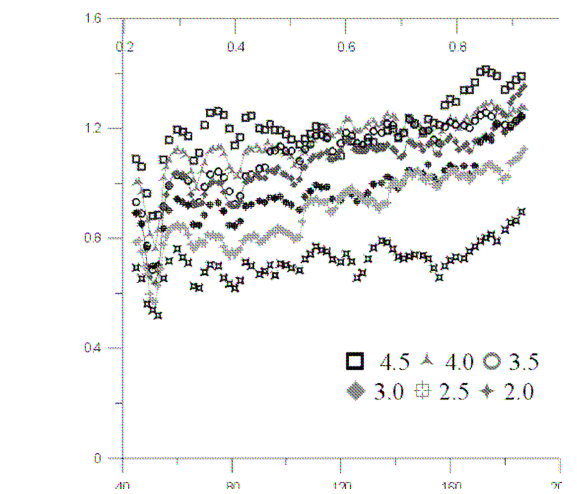

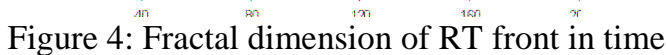
$(\mathrm{t} / \mathrm{T})$

Under laboratory conditions it is possible to obtain reliable velocities, vortices and discharges from image techniques. Volume fraction, velocity modulus or vorticity modulus may be measured with precision (in a $5000^{2}$ frame), we can thereby define the fractal dimension $D(i)$ as a function of the intensity $\mathrm{i}$ of the image. This dimension is usually calculated using: $D(i)=-\log (N(i)) / \log (e, i)$, Where $N(i)$ is the number of boxes of size e needed to cover the image contour of intensity $i$. We can decide, what is the range of scales, as well as the type of fit needed The algorithm operates dividing the 2D surface into smaller and smaller square boxes and counting the number of them which have values close to the level under study, This analysis can be made for the Scalar marker (passive or reactive), the Velocity or the Vorticity.
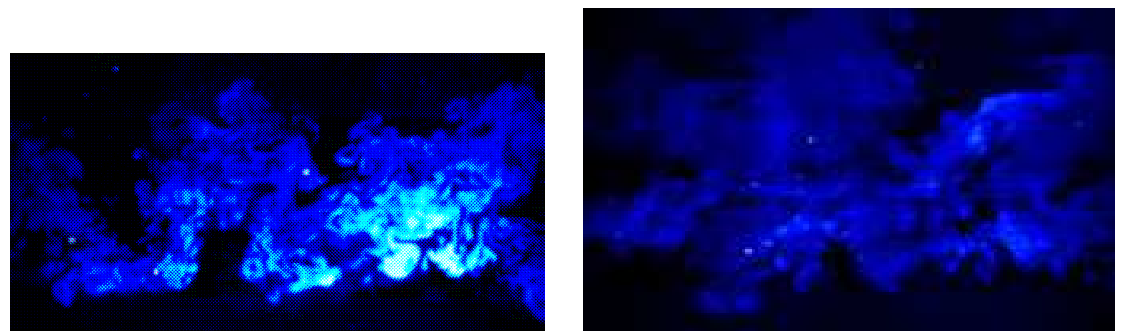

Figure 5: False color image of the details of the evolution of a mixing RT front, showing details of the blobs. (Dalziel and Redondo 2007).

In the case of isolated singularities, detected in the flow such as spiral vortices or dipoles, fractal approaches have been used previously: H. K. Moffat showed that the Fourier transform of the characteristic function of the one-dimensional spiral decays as $|\mathrm{k}|-2+1 /(1+\gamma)$, while J. C. Vassilicos and J. C. R. Hunt [12-14] remarked that the exponent in this power-law is directly related to the box dimension of the spiral since this box dimension is precisely $1 / 1+\gamma$. However, as they comment "The drawback of the properties based on Fourier analysis is that they give clear information only for one isolated singularity". Since Fourier analysis is non-local, the information concerning different local behaviors at different locations is completely mixed-up. This is an additional reason for rather using direct multifractal box counting methods and wavelet analysis when dealing in applications with experimental data, where many such complex interacting behaviors occur in high Reynolds number flows $[16,17]$. 

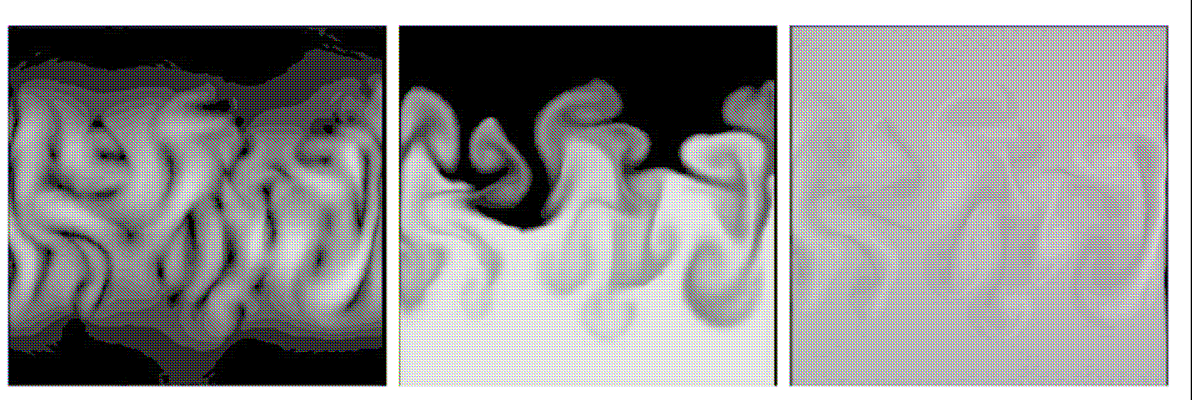

Figure 6: Comparison of Velocity modulus (left), Volume Fraction (center) and Vorticity (right) Contours
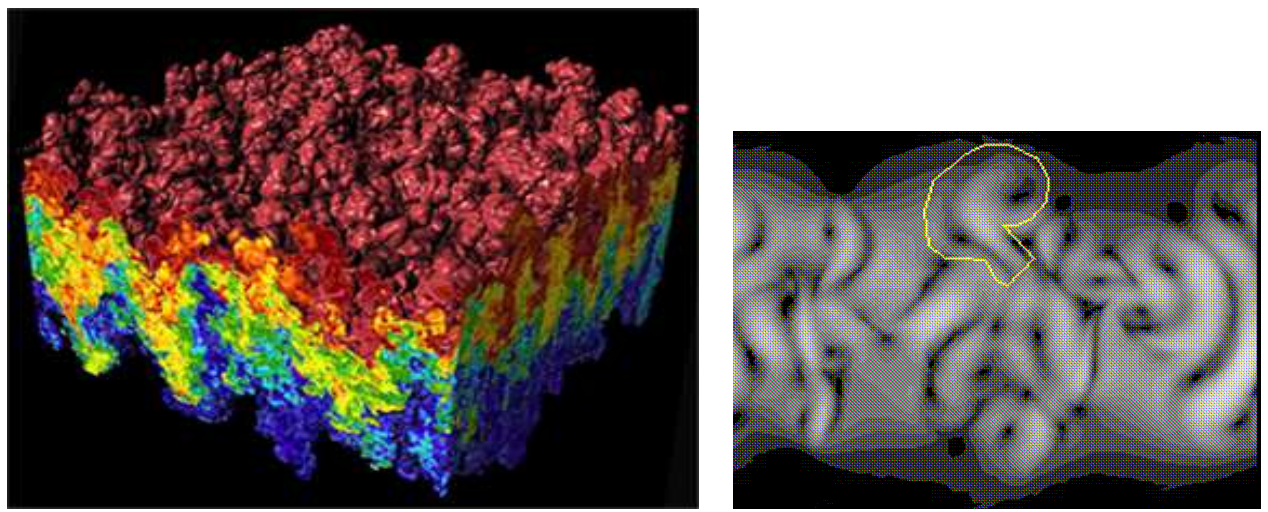

Figure 7: DNS simulation of Volume Fraction evolution (left) and single blob detail (right) [18,19].

In this case, for the Rayleigh Taylor flows under non-homogeneous conditions as shown in Figures 4-7 [6], experiments are able to obtain a better quantification of the intermittency using ESS in a 2D section of the flow such as that in figure 6. The relationship between intermittency, the fractal dimension at the sides and centres of the RT blobs is obtained using the sixth order structure function and the $\beta$-model. If $q$ is the order of the structure function, in this case $q=6$, because of the relation of turbulent dissipation (or production) to velocity fluctuations. In a similar way, the fourth order structure function may also be used as related directly to the kurtosis [15].

The multi fractal technique performed on the Rayleigh Taylor and Richtmyer Meshkov fronts, so far allows discrimination between the strong mixing areas and those weaker mixing regions that produce large smooth interfaces. We have analysed the Fractal and Multi-scale instabilities leading to mixing due to RT and RM instabilities using also wavelet and Neural techniques coupled with the multi fractal measurements as described in $[19,20]$ to aid the description of the self-similar structure within the mixing fronts. Elaboration of two-dimensional numerical simulation of evolution of the mixing region, under conditions used to drive the RT or the RM allow a parameter distribution of the incident compression wave used as boundary conditions are compared with two-dimensional numerical simulations. The simulations are expected to reflect accurately the gross features of the experimental RT and RM flows [10]. Concerning the fractal analysis, it is usual to identify different dynamic processes that influence the flow. The box-counting algorithm, able to detect the self-similar characteristics for different image intensity levels. This technique is used in the Numerical simulations for Velocity, Vorticity and Volume fraction images reflecting a physical aspect that is advected by the flows, we can thereby define the fractal dimension $D(i)$ as a function of the intensity $i$ of the relevant variable, the dimension is calculated using the box counting method. 


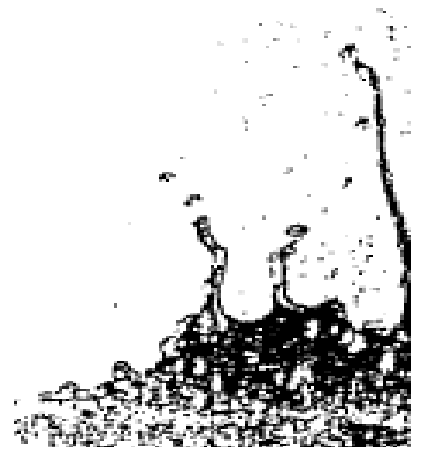

Figure 8: Visualization of a R-M Instability on a Hg-Air interface (Redondo et al. 2007)[8,14]

Further development of methods of simulating ICF layered targets in view of effects of compression and influence of change of parameters on the detailed process of mixing and stirring. The models will be required to successfully simulate the key statistical elements of the experimental results. Comparison with incompressible RT and with (compressible and incompressible) Richtmyer-Meshkov instability are related with fractal generated grid flows [18-20].
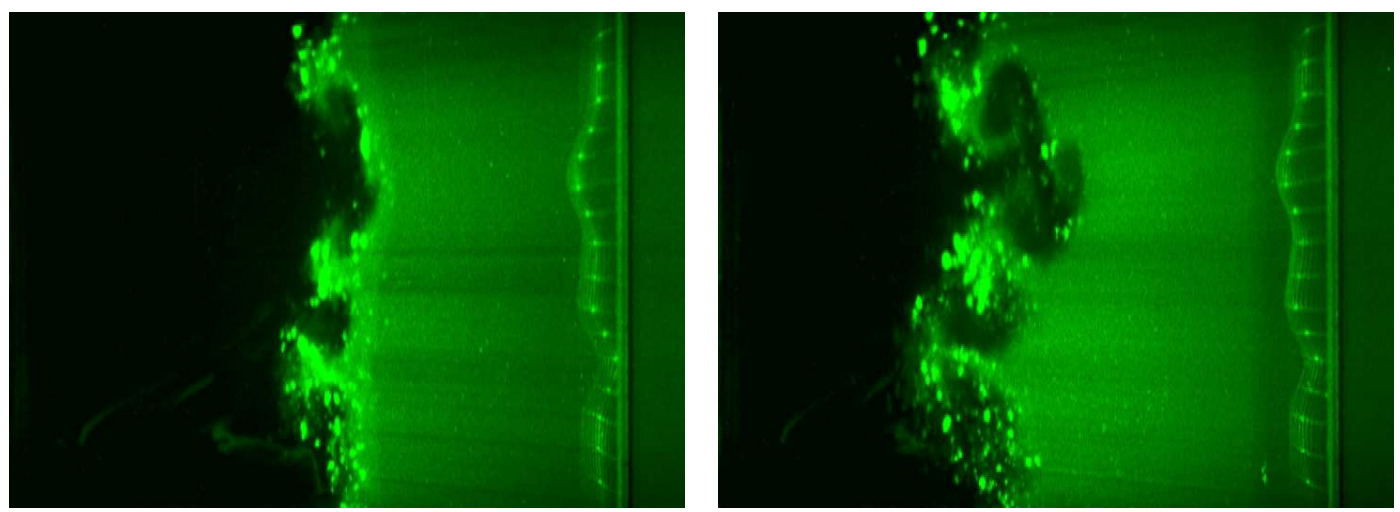

Figure 9: Visualizations of the front forced by a wavy membrane grid wakes (Houas et al. 2001)

Simulations performed at the BSC by Laizet, S. and Prof. Vassilicos with a numerical strategy to investigate fractal generated turbulence [12-17] were analysed with program ImaCalc to compare the evolution described by Redondo [18] and the variation of maximum fractal dimension and intermittency as a function of distance from the grid.

The effect of the largest topological structures of the wake or shock induced flows is to decrease the maximum fractal dimension and to reduce the range of intensity values where the flow is fractal (Figures $6,7)$. Of course surface tension or boundary and initial condition effects are fundamental as seen in the Mercury-Air interface of figure 8 . The scaling behavior is different for the different results detected with PIV and MFV. Most of the mixing taking place in the sides of the coherent evolving fields, but also depend on dominant structures, i.e. blobs and spikes, or imposed waves and membrane effects. The use of LES simulations and of KS synthetic turbulence was compared as reported by Redondo et al. [6, 20, 21]. Numerical simulations agree on average with the front advance experiments. But the higher order velocity measurements and local fractal measurements gives further insight on the different cascading processes that take place in the flow, mainly the tracer density spectra, the velocity, the vorticity spectra and the multi-fractal evolution. There has been relatively little effort to theoretically model the late-time asymptotic growth of the Richtmyer-Meshkov mixing layer from multi-mode perturbations. The selfsimilar mixing layer in unstable flows is predicted to grow as $h(t) \propto t^{\theta}$ with $\theta \in(1 / 3,1)[1,14]$. Buoyancy models have been formulated to describe $h(t)$ and as two-equation turbulence models can also be reduced to ordinary differential equations via the similarity transformation $\eta \propto(\mathrm{x} / \mathrm{t})^{\theta}$, the solutions give the 
exponent $\theta$ as a function of the model parameters and the mixing efficiency of the process [22, 23]. The high fractal descriptors may define the regions where most local mixing takes place.

\section{Discussion and Conclusions}

The relationship between stratification, intermittency, $D(R i)$ the fractal dimension of the flows and the turbulence energy spectral slope $b$, may be related [18-20]. For example the local measurements of dispersion, are related to the second order velocity moments, the skeweness and kurthosis, etc. are related to asymmetry and intermittency, and so on. Analysis from conditional statistics of the relationship between maximum fractal dimension and buoyancy $D(R i)$ [9] for stable flows have been confirmed and extended to unstable flows. Both the laboratory experiments and the 2D-3D aspects of the turbulence cascade are much more complicated than first believed. So use of a model comparing the corresponding relative scaling exponents estimated from more than two characteristic parameters $(D, b)$. Then it is possible to find values for these parameters that represent the empirical scaling exponents $D$ and $b$ obtained in a similar way as in [15]. In 2D flows, when D increases, the order smaller than 3 relative scaling exponents also increases (but for orders higher than 3, they decrease) not necessarily linearly. But for a certain value of $D$, when $b$ increases the behaviour of the relative scaling exponents is the opposite and non-linear [16].

It is not possible to perform an analysis independently on the initial conditions, neither for the RM nor for the RT flows. Therefore, the choice and classification of mixing descriptors has to consider the initial fields. The initial conditions are those of a $2 \mathrm{D}$ established flow with vortex shedding, providing a rectilinear configuration of the alternating vortex rows along the span of the flows. Baroclinic local production and vortex shedding keeps a large memory in the downstream mixing distance, but these features depend also on the plane of visualization. It is found that velocity component is soon organised according to well distinct structures such as those seen in figures 6,7 .

The contour plots of the vorticity structures in general are smoother and exhibit lower fractal the physical space present than those of scalar mixing or volume fraction. To extend the measurements to complex flows a novel pattern matching technique was developed. This technique utilises similar algorithms to those found by DigiFlow in PIV and in synthetic schlieren [10, 19].

For the shock tube mixing process, a pattern which may be part of the broken membrane used in the initial conditions [4, 22-24]. This pattern is then viewed at an angle from the side selected as a balance between the amplitude of the apparent movement of the structures compared before and after the mixing event (Figure 9). This allows the highly accurate pattern matching algorithms developed for synthetic schlieren to be used to produces a local velocity field. The boundary condition effects and the role of combining the topological 3D and 2D characteristics of the individual flows relate to the scale to scale turbulent direct and inverse cascades. Further work is needed that will probably set some limits to the forcing intermittent behaviour detected by [14-16] as well as producing some non-linear coupling between scales depending on the interaction between the forcing and the turbulent cascade.

An important consideration apparent in the evaluation of the intermittency and the multy-fractal dimensions. (for different levels of the marker) is that velocity, vorticity and volume-fraction or scalar concentration exhibit different scaling laws [24, 25]. This indicates the need for further comparisons to be made between numerical simulations with different initial conditions, and detailed experiments as Rayleigh-Taylor instability happens in many physical situations and is an important issue to understand the properties of mixtures and to relate them to some geometric (multi-fractal) and dynamical (mixing efficiency) properties of the flows.

\section{Acknowledgements}

Support from European Union through ISTC-1481 and INTAS- projects, and from MCT-FTN2001-2220 and DURSI XT2000-0052 local projects are acknowledged. PELNoT-ERCOFTAC and BEROTZA-CTT projects as well as MULTIFLOW have funded some of this work. We thank the help of the Barcelona Super Computer Marenostrum - BSC and the RES for funding Dr. Sylvan Layzet and Prof. J.C. Vassilicos in Barcelona. by the Spanish Ministry of Education and Science (projects CGL2009-12797C03-03 and CGL2012-37416-C04-02) Thanks are also due to Univ. Marseille and ERCOFTAC (SIG 14 and SIG4). 


\section{References}

[1] Redondo, J. M., Sanchez, M. A., Garriga, J., Castilla, R.: Convective and Rayleigh-Taylor Instabilities in Stratified Fluids. In Advances in Turbulence V. 428-434.1995, Springer Netherlands.

[2] Thorpe, S. A.: Turbulence and mixing in a Scottish loch. Philos. Trans. R. Soc. London, Ser. A, vol. 286: 1977. 125-181.

[3] Vindel, J. M., Yagüe, C. \& Redondo, J. M.: Structure function analysis and intermittency in the atmospheric boundary layer. Non Linear Proc. Geophys., vol. 15: 2008. 915-929

[4] Houas, L., Jourdan G.: Richtmyer-Meshkov instability induced turbulent mixing study in a new shock tube apparatus by a translated laser sheet technique. In: Proc 23rd Int. Symp.on Shock Wave, Fort Worth, Texas, USA, 22-27 July, 2001, ed. by F.K. Lu, 1141-1146

[5] Jacobs, W.G., Krivets V.V.: PLI Flow visualization of the nonlinear development and transition to turbulence of Richtmyer-Meshkov instability. In: Proc 23rd Int. Symp. on Shock Wave, Fort Worth, Texas, USA, 22-27 July, 2001, ed. by F.K. Lu, 1183-1189

[6] Redondo J. M. and Linden P. F.: Geometrical observations of turbulent density interfaces. in The Mathematics of Deforming Surfaces, edited by Dritschel D. G. and Perkins R. J. (Clarendon Press Oxford) 1996, 221.

[7] Redondo J. M.: Mixing efficiency of different kinds of turbulent processes and instabilities, Applications to the environment. in Turbulent Mixing in Geophysical Flows, edited by Linden P. F. and Redondo J. M. (CIMNE, Barcelona), 2002, 131-157.

[8] Castilla, R. \& Redondo, J. M.: Mixing Front Growth in RT and RM Instabilities. Proceedings of the Fourth International Workshop on the Physics of Compressible Turbulent Mixing, Cambridge, United Kingdom, edited by P. F. Linden, D. L. Youngs, and S. B. Dalziel, 1994, 11-31.

[9] Redondo, J. M.: Fractal models of density interfaces. In: IMA Conf. Ser. 13, Farge, M., Hunt, J.C.R., Vassilicos J. C. (eds.) Oxford: Clarendon Press/Elsevier, 1993; 353-370.

[10] Dalziel, S.D. and Redondo J.M.: New visualization and self-similar analysis in experimental turbulence studies. Models, Experiments and Computation in Turbulence. 2007. CIMNE, Barcelona.

[11] Tellez J., Gómez M., Russo B., Redondo J. M.: A simple measuring technique of surface flow velocity to analyze the behavior of velocity fields in hydraulic engineering applications. Geophysical Research Abstracts 2015 - EGU General Assembly 2015, Vol. 17.

[12] Malik N. A. \& Vassilicos J. C.: A Lagrangian model for turbulent dispersion with turbulent-like flow structure: comparison with direct numerical simulation for two-particle statistics. Phys. Fluids 11, 1999. 1572-1580.

[13] Moffat, H. K.: Simple topological aspects of turbulence velocity dynamics. In: Proc. IUTAM Symp. on Turbulence and Chaotic Phenomena in Fluids, ed. Tatsumi Amsterdam: Elsevier/North Holland, 1984, p. 223.

[14] Redondo J.M., J. Grau, A. Platonov, G. Garzon: Analisis multifractal de procesos autosimilares: imagenes de satelite e inestabilidades baroclinas. Rev. Int. Met. Num. Calc. Dis. Ing. 24, 2008, 2548, (in Spanish)

[15] Sekula E. and Redondo J.M.: The structure of turbulent jets, vortices and boundary layers: Laboratory and field observations. Il Nuovo Cimento C, vol 31, 5/6, 2008, 893-907.

[16] Nicolleau, F.C.G.A., Cambon, C., Redondo, J.M., Vassilicos, J.C., Reeks, M., Nowakowski, A.F. (Eds.): New Approaches in Modeling Multiphase Flows and Dispersion in Turbulence, Fractal Methods and Synthetic Turbulence. ERCOFTAC Series, Vol. 18. 2011, 250.

[17] Laizet, S., Lamballais, E., Vassilicos, J. C: A numerical strategy to combine high-order schemes, complex geometry and parallel computing for high resolution dns of fractal generated turbulence. Comput. Fluids 39(3), 2010, 471-484. 2010. 
[18] Redondo, J.M.: Topological Structures in Rotating Stratified Flows. Topical Problems of Fluid Mechanics 2003, J. Prihoda, K. Kozel. 2003, Prague.

[19] Redondo, J.M., Gonzalez-Nieto, P.L., Cano, J.L. and Garzon, G.A.: Mixing Efficiency across Rayleigh-Taylor and Richtmeyer-Meshkov Fronts. Open Journal of Fluid Dynamics, 5, 145-150. 2015.

[20] Redondo J.M., Sanchez M.A., Castilla R.: Vortical structures in stratified turbulent flows, in Turbulent Diffusion in the Environment, (Eds Redondo J. M. and Babiano A.) 2000, 113-120. FRAGMA, Madrid.

[21] Cantalapiedra I. R. and Redondo J. M.: Mixing in Geophysical Flows, edited by Redondo J. M. and Metais O. (International Center for Numerical Methods, CIMNE)1995, 127.

[22] V. B. Rozanov, I. G. Lcho. S.G. Zaitsev et al.: Experimental investigation of gravitational instability and turbulent mixing of stratified flows in an acceleration field in connection with the problem of inertial nuclear fusion, Preprint No. 56, FIAN, Moscow, 1990.

[23] Meshkov E. et al. (Eds.): Physics of Compressible Turbulent Mixing. Proc. 7th Int. Workshop. St. Petersburg, Russia, 1992.

[24] Redondo J.M.: Vertical microstructure and mixing in stratified flows. Advances in Turbulence VI. (Eds. S. Gavrilakis el al.) 1996, 605-608.

[25] Redondo J.M. and Cantalapiedra I.R.: Mixing in horizontally heterogeneous flows. Applied Scientific Research, 51, 1993, 217-222. 